\section{Declínio da cárie em um município da região noroeste do Estado de São Paulo, Brasil, no período de 1998 a 2004}

\author{
Declining caries rate in a municipality in \\ northwestern São Paulo State, Brazil, 1998-2004
}

Ronald Jefferson Martins 1 Cléa Adas Saliba Garbin 1 Artênio José Ísper Garbin 1 Suzely Adas Saliba Moimaz 1 Orlando Saliba 1

\author{
1 Faculdade \\ de Odontologia \\ de Araçatuba, \\ Universidade Estadual \\ Paulista, Araçatuba \\ Brasil. \\ Correspondência \\ R. J. Martins \\ Departamento de \\ Odontologia Infantil \\ e Social, Faculdade de \\ Odontologia de Araçatuba, \\ Universidade Estadual \\ Paulista. \\ R. José Bonifácio 1193, \\ Araçatuba, SP \\ 16015-050, Brasil. \\ rojema@terra.com.br
}

\begin{abstract}
This study analyzes the DMF and DEF indices, $\mathrm{SiC}$ index, and percentage of caries-free public schoolchildren in the municipality of Bilac, São Paulo State, Brazil, in 1998, 2000, 2002, and 2004. The same methodology was employed (WHO1997) in all the surveys. Kappa test was carried out at each stage, and the minimal and maximum inter-examiner agreement values obtained were 0.86 and 0.89 , respectively, while minimal intra-examiner agreement values were 0.91. DEF indices decreased slightly in the years 1998 and 2004. There was a continuous reduction in the DMF index, as well as the phenomenon of polarization at 12 years of age. The rate was 5.28 in 1998, decreasing to 4.11 in 2000, 3.47 in 2002, and 2.62 in 2004. Inversely, the proportion of caries-free 5-year-olds increased from $37.9 \%$ in 1998 to 40\% in 2000 and 2002 and 45.3\% in 2004. In conclusion, there has been a decrease in dental caries in 12 year-old public schoolchildren in the municipality.
\end{abstract}

Dental Caries; Health Surveys; Public Health Dentistry

\section{Introdução}

O conhecimento da situação epidemiológica da população é essencial para o planejamento e a execução de ações em saúde bucal, sendo o caminho correto de superação do atendimento indiscriminado da livre demanda, ou seja, do atendimento às pessoas que por sua própria iniciativa recorrem às unidades de tratamento disponíveis 1.

Em 1998, foi realizado o Levantamento Epidemiológico das Condições de Saúde Bucal da População do Estado de São Paulo, sob a coordenação da Faculdade de Saúde Pública, Universidade de São Paulo, objetivando o conhecimento das condições de saúde bucal da população para o redirecionamento das práticas de saúde e avaliação do impacto das medidas propostas. Abrangeu 24 Direções Regionais de Saúde (DIRs), representadas por 133 municípios e 89.114 participantes, distribuídos em faixas e grupos etários 2 .

O Município de Bilac foi um dos sorteados para participar desse levantamento. Outros três levantamentos foram realizados nos anos de 2000, 2002 e 2004. Esta periodicidade é consonante à Resolução SS-039, da Secretaria de Estado da Saúde de São Paulo 3.

No Brasil, com a introdução dos dentifrícios fluoretados no final da década de 80 , houve uma mudança no perfil epidemiológico de cárie dentária, ocorrendo a diminuição no ín- 
dice CPOD e aumento no percentual de indivíduos livres de cárie 2 .

Estudos em municípios brasileiros com e sem fluoretação das águas de abastecimento público não mostram diferença no índice CPOD e relatam tendências de declínio da cárie dentária 2,4,5,6.

Objetivou-se, neste trabalho, analisar o índice CPOD, ceod, Significant Caries Index e a porcentagem de crianças livres de cárie em escolares da rede pública de Bilac, no período de 1998 a 2004.

\section{Métodos}

O Município de Bilac, pertencente à DIR-VI (Araçatuba), localiza-se a $510 \mathrm{~km}$ da capital paulista, na região noroeste do Estado. Apresenta, segundo o Instituto Brasileiro de Geografia e Estatística, uma população estimada em 2004 de 6.410 habitantes, sendo $88 \%$ da população urbana, predominantemente adulta e paritária quanto ao sexo. Não existe sistema de fluoretação das águas do município.

A população estudada foi constituída por todos os escolares de 5 a 12 anos matriculados nas duas escolas da rede pública de ensino do Município de Bilac. Admitiu-se que determinadas idades corresponderiam a determinadas séries, sendo excluídas as crianças que, baseado na data de nascimento e dia do exame, estivessem fora da série correspondente. Também não foram examinadas as crianças cujos responsáveis não assinaram o termo de consentimento livre e esclarecido.

Adotou-se a mesma metodologia nos levantamentos realizados em 1998, 2000, $2002 \mathrm{e}$ 2004, tendo como base a quarta edição do Oral Health Surveys: Basic Methods da Organização Mundial da Saúde (OMS) de 19977 e o Caderno de Instruções do Levantamento Epidemiológico das Condições de Saúde Bucal da População do Estado de São Paulo de 1998 .

A equipe participante dos quatro estudos foi composta pelos quatro cirurgiões-dentistas pertencentes à rede municipal de saúde bucal como examinadores, e por auxiliares como anotadores e monitores.

Realizou-se a calibração dos examinadores antes de cada levantamento epidemiológico, visando a garantir a uniformidade de interpretação, compreensão e aplicação dos critérios. A técnica utilizada foi a do consenso, sem a preocupação de comparações com um examinador-padrão.

Aplicou-se nas calibrações o teste estatístico kappa com o objetivo de avaliar a concor- dância real interexaminadores. O valor mínimo observado foi de 0,86 e máximo de 0,89 , indicando ótima concordância.

$O$ instrumento utilizado na coleta de dados foi uma ficha adaptada com base na ficha simplificada da OMS. Os examinadores utilizaram um conjunto de instrumentais composto por um espelho bucal plano e uma sonda específica, desenvolvida pela OMS, conhecida como "sonda CPI" 8.

Adotaram-se as medidas vigentes de biossegurança visando à proteção da equipe pesquisadora e dos que se submeteram aos exames. Realizaram-se os exames nos pátios das escolas, com luminosidade natural.

Durante os levantamentos, realizaram-se exames em duplicata em $10 \%$ dos examinados, objetivando a detecção de possíveis mudanças no diagnóstico ao longo dos exames. $\mathrm{O}$ anotador organizou os reexames para que o examinador não fosse capaz de identificar os indivíduos que seriam reexaminados. A concordância de diagnóstico intra-examinador foi muito alta. $\mathrm{O}$ teste kappa foi calculado para cada um dos examinadores, atingindo um valor mínimo de 0,91.

Utilizaram-se os programas Epi Info, versão 6.04 (Centers for Disease Control and Prevention, Atlanta, Estados Unidos) e EPIBUCO para a apuração e análise dos dados.

Calculou-se o SiC Index ${ }^{9}$ na faixa etária de 12 anos para se ter uma visão completa da cárie dentária no caso de distribuições assimétricas.

\section{Resultados}

O número de crianças examinadas na faixa etária de 5 a 12 anos, nas duas escolas públicas do Município de Bilac, nos levantamentos de 1998, 2000, 2002 e 2004, foram respectivamente, 546, 573,668 e 782 , correspondendo a $63,7 \%, 69 \%$, $79,1 \%$ e $89,7 \%$ das crianças matriculadas (Tabela 1 ).

Observa-se em cada ano estudado uma correlação positiva entre o índice CPOD e a idade.

Nos anos de 1998 e 2004 verifica-se uma diminuição do índice em todas as idades. Na idade de 12 anos, base de comparação pela OMS, houve uma redução contínua no período de $50,38 \%$, passando de uma prevalência alta em 1998, para baixa em 2004 10. O declínio do índice foi testado utilizando-se o Mann-Whitney Test e o resultado mostrou diferença estatisticamente significante $(p<0,0001)$. Foi atingida em 2004 a meta da OMS para o ano 2000, que é CPOD igual ou menor a 311 (Tabela 2).

Os valores do $\mathrm{SiC}$ Index 9 (média CPOD para um terço do grupo com os maiores níveis da 
Número e porcentual de crianças de 5 a 12 anos examinadas nas escolas públicas de Bilac, São Paulo, Brasil, em 1998, 2000, 2002 e 2004

\begin{tabular}{|c|c|c|c|c|c|c|c|c|c|c|}
\hline \multirow{3}{*}{ Idade (anos) } & \multicolumn{10}{|c|}{ Ano } \\
\hline & \multicolumn{2}{|c|}{1998} & \multicolumn{2}{|c|}{2000} & \multicolumn{2}{|c|}{2002} & \multicolumn{2}{|c|}{2004} & \multicolumn{2}{|c|}{ Total } \\
\hline & $n$ & $\%$ & $n$ & $\%$ & $n$ & $\%$ & $n$ & $\%$ & $n$ & $\%$ \\
\hline 5 & 58 & 10,6 & 37 & 6,5 & 100 & 15,0 & 139 & 17,8 & 334 & 13,0 \\
\hline 6 & 62 & 11,3 & 95 & 16,6 & 80 & 12,0 & 77 & 9,8 & 314 & 12,2 \\
\hline 7 & 79 & 14,5 & 69 & 12,0 & 95 & 14,2 & 97 & 12,4 & 340 & 13,2 \\
\hline 8 & 74 & 13,6 & 73 & 12,7 & 112 & 16,8 & 104 & 13,3 & 363 & 14,1 \\
\hline 9 & 70 & 12,8 & 77 & 13,4 & 51 & 7,6 & 104 & 13,3 & 302 & 11,8 \\
\hline 10 & 60 & 11,0 & 87 & 15,2 & 89 & 13,3 & 108 & 13,8 & 344 & 13,4 \\
\hline 11 & 74 & 13,6 & 72 & 12,6 & 68 & 10,2 & 71 & 9,1 & 285 & 11,1 \\
\hline 12 & 69 & 12,6 & 63 & 11,0 & 73 & 10,9 & 82 & 10,5 & 287 & 11,2 \\
\hline Total & 546 & 100,0 & 573 & 100,0 & 668 & 100,0 & 782 & 100,0 & 2569 & 100,0 \\
\hline
\end{tabular}

Tabela 2

Índice CPOD e desvio padrão (DP) em crianças de 5 a 12 anos das escolas públicas de Bilac,

São Paulo, Brasil, em 1998, 2000, 2002 e 2004.

\begin{tabular}{|c|c|c|c|c|c|c|c|c|}
\hline \multirow[t]{3}{*}{ Idade (anos) } & \multicolumn{8}{|c|}{ Ano } \\
\hline & \multicolumn{2}{|c|}{1998} & \multicolumn{2}{|c|}{2000} & \multicolumn{2}{|c|}{2002} & \multicolumn{2}{|c|}{2004} \\
\hline & CPOD & DP & CPOD & $\mathrm{DP}$ & CPOD & DP & CPOD & $\mathrm{DP}$ \\
\hline 5 & 0,00 & 0,00 & 0,08 & 0,36 & 0,02 & 0,20 & 0,05 & 0,33 \\
\hline 6 & 0,21 & 0,66 & 0,07 & 0,33 & 0,11 & 0,42 & 0,17 & 0,59 \\
\hline 7 & 0,58 & 0,98 & 0,28 & 0,78 & 0,33 & 0,87 & 0,51 & 0,93 \\
\hline 8 & 1,34 & 1,60 & 0,25 & 0,66 & 0,58 & 1,13 & 0,70 & 1,17 \\
\hline 9 & 2,04 & 1,71 & 0,56 & 1,08 & 0,88 & 1,23 & 0,77 & 1,15 \\
\hline 10 & 3,15 & 1,88 & 1,89 & 1,93 & 1,21 & 1,31 & 1,16 & 1,57 \\
\hline 11 & 4,22 & 2,91 & 2,83 & 1,82 & 2,10 & 1,82 & 2,28 & 2,14 \\
\hline $12^{*}$ & 5,28 & 4,14 & 4,11 & 3,20 & 3,47 & 2,77 & 2,62 & 2,95 \\
\hline
\end{tabular}

* Mann-Whitney Test: $p<0,0001$.

doença) nos anos de 1998, 2000, 2002 e 2004 foram de 9,$9 ; 7,4 ; 6,1$ e 5,9, respectivamente. As médias nos dois terços do grupo com os menores índices de cárie foram 3,0; 2,4; 2,2 e 1,0 (Figura 1).

Na dentição decídua, o índice ceod diminuiu numericamente em todas as idades nos anos de 1998 e 2004 (Tabela 3).

A porcentagem de crianças livres de cárie $(\mathrm{CPOD}+\operatorname{ceod}=0)$ aumentou em todas as idades nos anos de 1998 e 2004, apesar disso, em 2004, na idade de 5 anos, ainda não foi atingida a meta da OMS de $50 \%$ para o ano 200011 . Foi realizado teste de proporção e o resultado mostrou que a diferença não foi estatisticamente significante $(\mathrm{p}=0,34)$ (Tabela 4$)$.

\section{Discussão}

Durante as últimas duas décadas houve um marcante declínio da experiência de cárie em crianças e jovens, e uma proporção crescente de crianças livres de cárie em muitos países industrializados do mundo 12,13,14. A causa com maior evidência científica desse fenômeno é a adição de flúor nos dentifrícios e as mais bem justificadas tecnicamente são: as mudanças no consumo de açúcar (menor consumo de açúcar pelas crianças), mudanças no diagnóstico da cárie e melhorias nas condições sócio-econômicas 13. Na Finlândia, Hausen et al. 15, ao compararem o índice de cárie nos dentes decí- 
Figura 1

Comparação entre a média CPOD nos dois terços da população com os menores índices de cárie e a média CPOD de um terço da população com os maiores índices de cárie (SiC Index), na faixa etária de 12 anos, nos anos de 1998, 2000, 2002 e 2004. Bilac, São Paulo, Brasil.

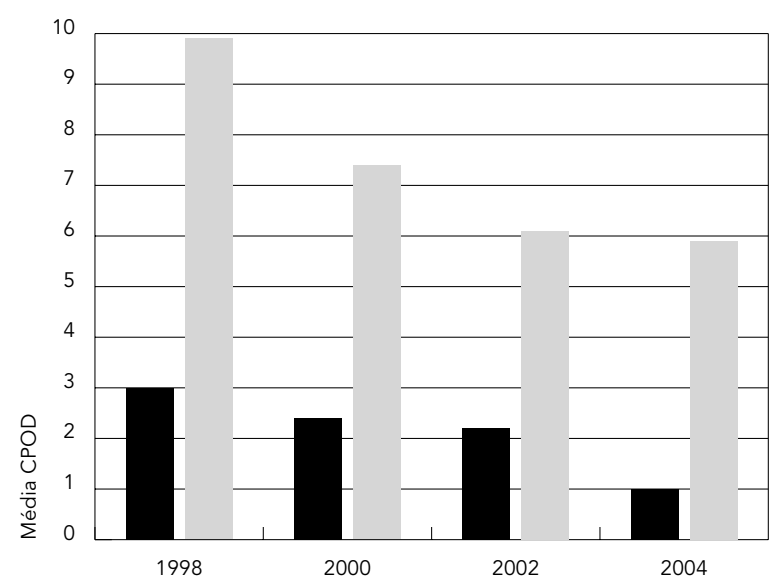

duos de crianças de diferentes classes sociais residentes em áreas com alto e baixo teor de flúor na água, verificaram que em classe sócioeconômica elevada o índice de cárie nos dentes decíduos é baixo, tanto naquelas beneficiadas e não pelos fluoretos.

Bastos et al. 16 atribuem ao binômio fluoretação de águas de abastecimento público e uso de dentifrícios fluoretados a modificação do perfil epidemiológico da cárie dentária no Brasil. Segundo Nadanovsky 13, a água fluoretada parece oferecer um benefício adicional, mesmo quando as pastas de dente contêm flúor.

Três grandes estudos epidemiológicos de abrangência nacional propiciaram a análise dos padrões de saúde bucal no país, todos limitados à zona urbana. O primeiro foi realizado em 1986 pelo Ministério da Saúde envolvendo 16 capitais de Estados, onde foram examinadas 15.009 crianças na faixa entre 6 e 12 anos. O segundo foi desenvolvido pelo Serviço Social da Indústria, em 1993, com recursos fornecidos pelo Ministério da Saúde envolvendo cidades das capitais e interior de 23 Estados, sendo examinadas 78.293 crianças na faixa entre 7 e 14 anos. O terceiro realizado em 1996 pela Fundação Nacional de Saúde, em parceria com a Associação Brasileira de Odontologia nacional, Associação Paulista de Cirurgiões Dentistas de São Paulo, Conselho Federal de Odontologia e Secretarias Estaduais de Saúde englo- bou as capitais dos Estados e o Distrito Federal, sendo examinadas 30.240 crianças na faixa entre 6 e 12 anos 2,16,17.

Apesar de existirem dúvidas sobre a validade e a confiabilidade desses achados em razão de diferentes metodologias e de critérios de diagnóstico utilizados 18 , pode-se verificar uma redução considerável no padrão de cárie dentária no período que se estende de 1986 a 1996, em todas as faixas etárias 2,17. Em 1996, o CPOD aos 12 anos apresentou um valor de 6,65, indicando, de acordo com a escala da OMS, uma prevalência muito alta, na época constituía o terceiro pior índice do mundo 17. Em 1993, a média geral era de 4,84 representando uma queda de $27,2 \%$ em relação ao dado de sete anos antes, passando a uma prevalência alta. Em 1996 a média foi de 3,06, ficando muito próxima da meta estabelecida para o ano 2000 pela OMS/World Dental Federation 11, passando a uma prevalência média. Esses estudos sugerem uma redução de $53,2 \%$ ao longo desses anos 18 .

Não estão claras quais as reais causas do declínio nacional e se a redução é realmente dessa magnitude. Roncalli 17 acredita não ser um fator isolado, considerando importante o aumento do uso de pasta fluoretada e a reorganização da prática odontológica pública, com maior ênfase nas atividades de promoção da saúde. Para Narvai et al. 19, a fluoretação das águas de abastecimento público, a adição de compostos fluoretados aos dentifrícios e a descentralização do sistema de saúde brasileiro são fatores que devem ser considerados para compreender o processo de declínio na experiência de cárie em dentes permanentes de escolares brasileiros durante o final do século XX.

Além dos dados nacionais mostrarem esse declínio, diversos dados obtidos isoladamente em vários estados e municípios brasileiros mostram a mesma tendência de queda 16,17,18,19,20,21.

O Levantamento das Condições de Saúde Bucal da População do Estado de São Paulo realizado em 1998, mostrou que o CPOD médio aos 12 anos passou de 6,47 em 1986 para 3,72 em 1998 19,22.

No Município de Bilac está ocorrendo declínio dos índices CPOD e ceod e aumento da porcentagem de crianças livres de cárie (CPOD + ceod $=0$ ), apesar de o município não apresentar sistema de fluoretação das águas de abastecimento público. Sales-Peres 2 analisou o perfil epidemiológico de cárie dentária na região centro-oeste do Estado de São Paulo e verificou que não houve diferença significativa entre o CPOD de municípios de mesmo porte, com ou sem flúor na água. As regiões fluoretadas e não-fluo- 
Índice ceod e desvio padrão (DP) em crianças de 5 a 12 anos das escolas públicas de Bilac, São Paulo, Brasil, em 1998, 2000, 2002 e 2004.

\begin{tabular}{|c|c|c|c|c|c|c|c|c|}
\hline \multirow[t]{3}{*}{ Idade (anos) } & \multicolumn{8}{|c|}{ Ano } \\
\hline & \multicolumn{2}{|c|}{1998} & \multicolumn{2}{|c|}{2000} & \multicolumn{2}{|c|}{2002} & \multicolumn{2}{|c|}{2004} \\
\hline & ceod & DP & ceod & $\mathrm{DP}$ & ceod & $\mathrm{DP}$ & ceod & DP \\
\hline 5 & 2,93 & 3,28 & 2,70 & 3,17 & 2,53 & 3,22 & 2,84 & 3,66 \\
\hline 6 & 3,31 & 3,47 & 3,56 & 3,34 & 3,05 & 3,30 & 2,88 & 3,24 \\
\hline 7 & 4,23 & 3,39 & 3,52 & 3,12 & 3,22 & 3,00 & 3,27 & 3,03 \\
\hline 8 & 3,82 & 3,03 & 3,29 & 2,69 & 2,80 & 2,77 & 2,54 & 2,42 \\
\hline 9 & 3,04 & 2,42 & 2,79 & 2,76 & 2,27 & 2,07 & 2,25 & 2,17 \\
\hline 10 & 1,80 & 2,05 & 1,53 & 2,13 & 1,58 & 2,09 & 1,76 & 2,10 \\
\hline 11 & 0,74 & 1,36 & 0,94 & 1,59 & 0,87 & 1,51 & 0,61 & 1,06 \\
\hline 12 & 0,33 & 0,90 & 0,32 & 0,88 & 0,47 & 1,21 & 0,15 & 0,45 \\
\hline
\end{tabular}

\section{Tabela 4}

Número e porcentual de crianças de 5 a 12 anos livres de cárie, das escolas públicas de Bilac,

São Paulo, Brasil, em 1998, 2000, 2002 e 2004.

\begin{tabular}{|c|c|c|c|c|c|c|c|c|}
\hline \multirow[t]{3}{*}{ Idade (anos) } & \multicolumn{8}{|c|}{ Ano } \\
\hline & \multicolumn{2}{|c|}{1998} & \multicolumn{2}{|c|}{2000} & \multicolumn{2}{|c|}{2002} & \multicolumn{2}{|c|}{2004} \\
\hline & $n$ & $\%$ & $n$ & $\%$ & $\mathrm{n}$ & $\%$ & $\mathrm{n}$ & $\%$ \\
\hline $5^{*}$ & 22 & 37,9 & 15 & 40,5 & 40 & 40,0 & 63 & 45,3 \\
\hline 6 & 20 & 32,3 & 22 & 23,2 & 27 & 33,8 & 25 & 32,5 \\
\hline 7 & 15 & 19,0 & 17 & 24,6 & 23 & 24,2 & 24 & 24,7 \\
\hline 8 & 16 & 21,6 & 16 & 21,9 & 31 & 27,7 & 27 & 26,0 \\
\hline 9 & 6 & 8,6 & 16 & 20,8 & 7 & 13,7 & 23 & 22,1 \\
\hline 10 & 1 & 1,7 & 21 & 24,1 & 21 & 23,6 & 31 & 28,7 \\
\hline 11 & 2 & 2,7 & 10 & 13,9 & 18 & 26,5 & 13 & 18,3 \\
\hline 12 & 4 & 5,8 & 6 & 9,5 & 11 & 15,1 & 21 & 25,6 \\
\hline
\end{tabular}

* Qui-quadrado: $p=0,34$ (ns).

retadas apresentam o fenômeno da "convergência”, que é a exposição dos indivíduos aos diferentes veículos com flúor. Muitos produtos consumidos pela população apresentam flúor em sua composição, como sucos em pó, leite em pó, achocolatados, comidas, antibióticos fluoretados etc. Outro fator é a presença do efeito "halo", que ocorre pelo consumo de produtos manufaturados produzidos em regiões fluoretadas e consumidos em regiões não-fluoretadas.

A Resolução SS-039 regulamentou um tipo de procedimento odontológico identificado como "procedimento coletivo" ${ }^{3}$. É um conjunto de procedimentos de promoção e prevenção em saúde bucal, de baixa complexidade e que dispensam equipamentos odontológicos. Den- tre eles, o município realiza somente os bochechos fluoretados, que podem influir no declínio da cárie dentária, embora segundo Disney et al. 23,24 a cárie diminuiu na presença ou na ausência de programas de bochecho de flúor e de outras formas de aplicação tópica de flúor.

Um dos parâmetros mais importantes usados como indicador de saúde bucal refere-se ao número e porcentual de crianças livres de cárie 16. O declínio da cárie dentária observado na quase totalidade dos países desenvolvidos e em parte dos chamados subdesenvolvidos ou em desenvolvimento, tem permitido observar o fenômeno da "polarização" da cárie dentária, onde a mesma não está mais distribuída uniformemente entre as crianças, concentrando- 
se em pequenos grupos populacionais grande número de dentes cariados, perdidos e restaurados e caracterizando-se pelo surgimento de um percentual cada vez maior de crianças livres de cárie dentária 2,21,22.

Segundo Nadanovsky 13, a polarização para a cárie dentária é aquela em que cerca de $70 \%$ dos dentes afetados pela doença estão em $30 \%$ das crianças. Em Bilac, acompanhando o decréscimo do índice CPOD, o porcentual de crianças livres de cárie dentária aumentou, indicando uma tendência à polarização, fenô- meno muito comum em situações de baixa prevalência e severidade 17 .

\section{Conclusão}

Está ocorrendo a redução estatisticamente significativa da cárie dentária e o fenômeno da polarização na faixa etária de 12 anos. Também o aumento do percentual de crianças livres de cárie (não-significativo estatisticamente), entre os escolares do ensino público do município.

\section{Resumo}

O objetivo do estudo foi analisar os índices CPOD, ceod, SiC Index e a porcentagem de crianças livres de cárie, em escolares de 5 a 12 anos da rede pública do Município de Bilac, São Paulo, Brasil, em estudos realizados nos anos de 1998, 2000, 2002 e 2004. Utilizouse a mesma metodologia (OMS-1997) em todos os levantamentos. O teste estatístico kappa foi calculado a cada estudo, obtendo-se o valor de concordância interexaminadores mínimo de 0,86 e máximo de 0,89, e intra-examinador mínimo de 0,91. O índice ceod diminuiu pouco nos anos de 1998 e 2004. Houve uma redução contínua do índice CPOD aos 12 anos, passando de 5,28 em 1998 para 4,11 em 2000, 3,47 em 2002 e 2,62 em 2004, e o fenômeno da polarização. $\mathrm{Na}$ proporção inversa, a porcentagem de crianças com 5 anos livres de cárie aumentou de 37,9\% em 1998 para 40\% em 2000 e 2002, e 45,3\% em 2004. Conclui-se que está ocorrendo a redução da cárie dentária na faixa etária de 12 anos entre escolares do ensino público do município.

Cárie Dentária; Levantamentos Epidemiológicos; Odontologia em Saúde Pública

\section{Colaboradores}

R. J. Martins participou da coleta e tabulação dos dados, discussão dos resultados, elaboração da conclusão e redação do texto. C. A. S. Garbin e A. J. Í. Garbin executaram correções no manuscrito e participaram da discussão dos resultados. S. A. S. Moimaz participou da discussão dos resultados. O. Saliba realizou a análise estatística do trabalho.

\section{Agradecimentos}

Os autores agradecem a todos os responsáveis pelas crianças que autorizaram a realização dos exames, aos cirurgiões-dentistas e auxiliares que contribuíram para a realização da pesquisa e à Prefeitura $\mathrm{Mu}$ nicipal de Bilac pelo apoio recebido. 


\section{Referências}

1. Pinto VG. Saúde bucal: odontologia social e preventiva. 4a Ed. São Paulo: Editora Santos; 2000.

2. Sales-Peres SHC. Perfil epidemiológico de cárie dentária, em cidades fluoretadas e não fluoretadas, na região centro-oeste do Estado de São Paulo [Dissertação de Mestrado]. Bauru: Universidade de São Paulo; 2001.

3. São Paulo. Resolução SS-039. Dispõe sobre as rotinas visando ao acompanhamento dos procedimentos coletivos das ações básicas em odontologia, nos serviços de saúde integrantes do SUS-SP. Diário Oficial do Estado 1999; 17 mar.

4. Dini EL, Holt RD, Bedi R. Comparison of two indices of caries patterns in 3-6 year old Brazilian children from area with different fluoridation histories. Int Dent J 1998; 48:378-85.

5. Gushi LL, Soares MC, Forni TIB, Vieira V, Wada RS, Sousa MLR. Cárie dentária em adolescentes de 15 a 19 anos de idade no Estado de São Paulo, Brasil, 2002. Cad Saúde Pública 2005; 21:1383-91.

6. Pereira AC, Da Cunha FL, Meneghim MC, Werner CW. Dental caries and fluorosis prevalence study in a nonfluoridated Brazilian community: trend analysis and toothpaste association. J Dent Child 2000; 67:132-5.

7. World Health Organization. Oral health surveys: basic methods. $4^{\text {th }}$ Ed. Geneva: World Health Organization; 1997.

8. Faculdade de Saúde Pública, Universidade de São Paulo/Secretaria de Estado da Saúde de São Paulo. Caderno de instruções do levantamento das condições de saúde bucal - Estado de São Paulo. São Paulo: Faculdade de Saúde Pública, Universidade de São Paulo/Secretaria de Estado da Saúde de São Paulo; 1998.

9. World Health Organization. Significant caries index - SiC. http://www.whocollab.od.mah.se/ expl/sic.html (acessado em 10/Set/2005).

10. Rêgo DM, Silva EM, Costa, PAP. Epidemiologia das doenças bucais. In: Universidade Federal do Rio Grande do Norte, organizador. Odontologia preventiva e social. Natal: Editora da UFRN; 1997. p. 93-113.

11. Secretaria de Estado da Saúde. A organização das ações de saúde bucal na atenção básica. Uma proposta para o SUS São Paulo. São Paulo: Centro Técnico de Saúde Bucal; 2001.

12. Bowen WH, Tabak LA. Cariologia para a década de 90. São Paulo: Editora Santos; 1995.

13. Nadanovsky P. O declínio da cárie. In: Pinto VG, organizador. Saúde bucal coletiva. 4a Ed. São Paulo: Editora Santos; 2000. p. 341-51.

14. Thylstrup A, Fejerskov O. Cariologia clínica. 2a Ed. São Paulo: Editora Santos; 1995.
15. Hausen H, Milen A, Heinonen OP, Paunio I. Caries in primary dentition and social class in high and low fluoride areas. Community Dent Oral Epidemiol 1982; 10:33-6.

16. Bastos RS, Bijella VT, Bastos JRM, Buzalaf MAR. Declínio de cárie dentária e incremento no percentual de escolares, de 12 anos de idade, livres da doença, em Bauru, São Paulo, entre 1976 e 1995. Rev Fac Odontol Bauru 2002; 10:75-80.

17. Roncalli AG. Perfil epidemiológico de saúde bucal no Brasil 1986-1996. http://www.unb.br/fs / sbc/documentos/epidemiologia/epi_bra.pdf (acessado em 20/Jan/2005).

18. Freysleben GR, Peres MAA, Marcenes W. Prevalência de cárie e CPO-D médio em escolares de doze a treze anos de idade nos anos de 1971 e 1997, região Sul, Brasil. Rev Saúde Pública 2000; 34:304-8.

19. Narvai PC, Frazão P, Castellanos RA. Declínio na experiência de cárie em dentes permanentes de escolares brasileiros no final do século XX. Odontologia e Sociedade 1999; 1:25-9.

20. Bastos JLD, Nomura LH, Peres MA. Tendência de cárie dentária em escolares de 12 e 13 anos de idade de uma mesma escola no período de 1971 a 2002, em Florianópolis, Santa Catarina, Brasil. Cad Saúde Pública 2004; 20:117-22.

21. Narvai PC, Castellanos RA, Frazão P. Prevalência de cárie em dentes permanentes de escolares do município de São Paulo, SP, 1970-1996. Rev Saúde Pública 2000; 34:196-200.

22. Narvai PC, Forni TIB, Junqueira SR, Cury JA, Castellanos RA, Soares, MC. Uso de produtos fluorados conforme o risco de cárie dentária: uma revisão crítica. http://www.apcd.org.br/Biblioteca/ Revista/2002/mar_abr/101.asp (acessado em 19/ Jan/2005).

23. Disney JA, Graves RC, Stamm JW, Bohannan HM, Abernathy JR. Comparative effects of a 4-year-fluoride mouthrinse program on high and low caries forming grade 1 children. Community Dent Oral Epidemiol 1989; 17:139-43.

24. Disney JA, Bohannan HM, Klein SP, Bell RM. A case study in contesting the conventional wisdom: school-based fluoride mouthrinse programs in the USA. Community Dent Oral Epidemiol 1990; 18:46-54.

Recebido em 12/Abr/2005

Versão final reapresentada em 29/Set/2005

Aprovado em 04/Out/2005 